\title{
Prevalence of diabetes complications in people with type 2 diabetes mellitus and its association with baseline characteristics in the multinational $A_{1}$ chieve study
}

Leon Litwak ${ }^{1 *+}$, Su-Yen Goh ${ }^{2 \dagger}$, Zanariah Hussein ${ }^{3+}$, Rachid Malek ${ }^{4+}$, Vinay Prusty ${ }^{5+}$ and Mohammad E Khamseh ${ }^{6+}$

\begin{abstract}
Background: Current International Diabetes Federation guidelines recommend a target $\mathrm{HbA}_{1 c}<7.0 \%$, but many people with diabetes worldwide find this difficult to achieve, increasing their risk of developing complications. This publication examines the prevalence of diabetes complications and its association with baseline characteristics in people with type 2 diabetes who participated in the $A_{1}$ chieve study.

Methods: $A_{1}$ chieve was a 24-week, multinational, open-label, observational study of 66,726 people with type 2 diabetes who had begun using biphasic insulin aspart 30, insulin aspart, or insulin detemir in routine clinical care. Participants were enrolled from 28 countries across four continents (Asia, Africa, Europe and South America). Baseline measurements of disease characteristics included: glycated haemoglobin ( $\left.\mathrm{HbA}_{1 c}\right)$, fasting $(\mathrm{FPG})$ and post-prandial plasma glucose (PPG), high- and low-density lipoprotein cholesterol (H- or LDL-C), systolic blood pressure (SBP), and body mass index (BMI). Data on complications and use of vascular disease preventative drugs were collected.

Results: Complication rates were high (27.2\% had macrovascular complications and 53.5\% had microvascular complications), particularly in Russia, and use of vascular disease preventative drugs was lower than expected. Age, BMI, diabetes duration, LDL-C, and SBP were positively associated, and HDL-C negatively associated, with macro- and microvascular complications (all $\mathrm{p}<0.05$ ). $\mathrm{HbA}_{1 c}$ and FPG were negatively associated with macrovascular complications (both $p<0.05$ ), which may be linked to the cross-sectional study design.

Conclusions: These results suggest a worldwide failure to achieve glycaemic targets. Better diabetes management with earlier initiation and optimisation of insulin regimens (e.g., with insulin analogues in the $A_{1}$ chieve population) may reduce the prevalence of vascular complications, improve the lives of people with diabetes and reduce the burden on healthcare systems.
\end{abstract}

Keywords: $A_{1}$ chieve study, Macro- and microvascular complications, Type 2 diabetes, Insulin analogues

\section{Introduction}

Effective management of diabetes requires sustained glycaemic control over many years to lower the risk of macro- and microvascular complications in people with diabetes. The UK Prospective Diabetes Study (UKPDS) found that every $1 \%$ reduction in glycated haemoglobin

\footnotetext{
*Correspondence: leon.litwak@hospitalitaliano.org.ar

${ }^{\dagger}$ Equal contributors

'Endocrinology and Nuclear Medicine Unit, Diabetes and Metabolism

Section, Hospital Italiano de Buenos Aires, Buenos Aires, Argentina

Full list of author information is available at the end of the article
}

$\left(\mathrm{HbA}_{1 \mathrm{c}}\right)$ was associated with a $37 \%$ decrease in microvascular disease and a $14 \%$ reduction in myocardial infarction (MI) [1]. Results of a 10-year follow-up study found that people with type 2 diabetes who maintain good glycaemic control experience benefits many years later, including lower rates of MI and diabetes-related death [2].

Accordingly, International Diabetes Federation (IDF) and joint American Diabetes Association (ADA)/European Association for the Study of Diabetes (EASD) guidance 
recommend a target $\mathrm{HbA}_{1 \mathrm{c}}$ of $<7.0 \%$ [3,4]. These guidelines highlight the need to review and to modify therapy regimens when $\mathrm{HbA}_{1 \mathrm{c}}$ goals can no longer be maintained $[4,5]$, which may include initiation and intensification of insulin therapy. Current diabetes guidelines also emphasise that cardiovascular disease risk reduction should be a focus of therapy [4-6]. However, most people with diabetes find it difficult to achieve $\mathrm{HbA}_{1 \mathrm{c}}$ levels $<7.0 \%$ [7-10], with $<40 \%$ of people with diabetes in a USA national survey reaching this target [9]. Moreover, in developing regions, including Eastern Europe, Asia, Latin America, and Africa, it has been shown that $22 \%$ and $36 \%$ of people with type 1 diabetes and type 2 diabetes, respectively, have never had their $\mathrm{HbA}_{1 \mathrm{c}}$ measured, and of those with available $\mathrm{HbA}_{1 \mathrm{c}}$ values, only $25 \%$ of people with type 1 diabetes and $36 \%$ of people with type 2 diabetes had $\mathrm{HbA}_{1 \mathrm{c}}$ levels $<7.0 \%$ [11]. This may increase the likelihood of progression of diabetes complications and of developing complications in later life. Reasons for the difficulty in achieving target $\mathrm{HbA}_{1 \mathrm{c}}$ levels may include problems adhering to complex oral and injectable therapies, concerns about insulin tolerability, psychological insulin resistance, fears about the risk of hypoglycaemia, and weight gain after insulin initiation [12-16].

More data are needed to evaluate glycaemic control and guideline adherence by physicians in real-life clinical practice in different geographical regions. These data can be used to assess whether glycaemic control, or any other factors, predict the establishment of diabetesrelated complications in various ethnic groups.

$\mathrm{A}_{1}$ chieve was a multinational, open-label, observational study of people with type 2 diabetes to assess clinical safety and effectiveness of biphasic insulin aspart 30 (NovoMix $\left.{ }^{\oplus} 30\right)$, insulin aspart $\left(\right.$ NovoRapid $\left.^{\circ}\right)$, or insulin detemir (Levemir ${ }^{\circ}$ ) with or without oral glucose-lowering drugs (OGLDs) in routine clinical care [17]. Participants were enrolled from 28 countries in Asia, Africa, South America and Europe [17]. In this report, diabetes complications data at baseline were assessed by geographical region and the relationship of these complications to other variables (e.g., patient and disease characteristics) was assessed by correlation analyses.

\section{Methods}

This was a 24-week international prospective, multicentre, open-label, non-interventional study in people with type 2 diabetes mellitus who had started biphasic insulin aspart 30, basal insulin detemir or bolus insulin aspart. These study drugs could be given alone or in combination with other medications that the participants were already taking. Baseline data, before participants received any of these insulin analogues or within 4 weeks of receiving insulin analogues, are reported in this analysis. Participants were evaluated by general physicians or diabetes specialists at the baseline visit, an interim visit approximately 12 weeks after the baseline visit, and a final visit approximately 24 weeks after the baseline visit.

The participants were enrolled in the study between January 2009 and June 2010. The 28 countries were grouped into seven geographical regions: China; South Asia (Bangladesh, India, and Pakistan); East Asia (Indonesia, Korea, Malaysia, Philippines, Singapore, and Taiwan); North Africa (Algeria, Morocco, Tunisia, and Libya); Middle East/Gulf (Egypt, Iran, Jordan, Turkey, Bahrain, Kuwait, Oman, Qatar, Saudi Arabia, United Arab Emirates, and Yemen); South America (Argentina and Mexico); and Europe (Russia).

Inclusion and exclusion criteria were kept to a minimum in order to reflect routine clinical practice as closely as possible. Selection of patients and choice of insulin regimen were at the discretion of the physician based on their clinical judgement. Exclusion criteria were: patients treated with biphasic insulin aspart 30, insulin detemir or insulin aspart (alone or in combination) for more than 4 weeks before enrolment in the study; patients previously enrolled in the study; patients with hypersensitivity to biphasic insulin aspart 30, insulin detemir or insulin aspart or to any of its excipients; and women who were pregnant, breast-feeding or had the intention of becoming pregnant within the next six months.

All local requirements for Health Authorities and Ethics Committee approvals were obtained, if applicable. The study was conducted in accordance with the Declaration of Helsinki [18] and the Guidelines for Good Pharmacoepidemiology Practice [19].

\section{Assessments}

Only baseline measurements are reported in this publication, which included patient characteristics, disease characteristics (metabolic control), diabetic macro- and microvascular complications, and use of vascular disease preventative drugs. These data were tabulated by total study population and by geographical region.

Patient characteristics assessed at baseline included: age, sex, diabetes duration, and body mass index (BMI). Disease-related characteristics assessed at baseline included: $\mathrm{HbA}_{1 \mathrm{c}}$, fasting plasma glucose (FPG) before breakfast, post-prandial plasma glucose (PPG) after breakfast, total cholesterol (TC), triglycerides (TG), creatinine, high-density lipoprotein cholesterol (HDL-C), low-density lipoprotein cholesterol (LDL-C), systolic blood pressure (SBP), and quality of life (QoL). QoL was assessed using the standardised $\mathrm{EQ}-5 \mathrm{D}^{\mathrm{Tm}}$ where $100=$ best imaginable health and $0=$ worst imaginable health [20].

Since this is an open label, observational study, all complications data were collected from available medical 
records or memory, with no additional clinical assessments performed. Data were collected for the following macrovascular complications based on the physician's clinical judgement: MI, angina, peripheral vascular disease, stroke, heart failure, atrial fibrillation, and left ventricular hypertrophy. Data were collected for the following microvascular complications: renal disease (microalbuminuria, gross proteinuria, end-stage renal disease), eye problems (background diabetic retinopathy, proliferative diabetic retinopathy, severe vision loss, macular oedema, and cataract), foot ulcer (uninfected ulcer, infected ulcer, healed ulcer, and history of amputation), and diabetic neuropathy. Data collected on use of vascular disease preventative drugs included use of renin-angiotensin system (RAS) blockers, aspirin and statins.

\section{Statistical analysis}

The sample size calculation was based on the number of patients $(20,000)$ required to detect, with at least 95\% confidence, serious adverse drug reactions (SADRs) including major hypoglycaemia events, with an incidence of 15 events 100,000 (0.015\%) for a single modern insulin during the 6 months of the study. Assuming equal distribution of patients among the three modern insulins, the estimated sample size was 60,000 patients.

The influence of variables on vascular complications was evaluated using a univariate logistical regression model as a first step, run separately for macrovascular and microvascular complications. Patient and disease characteristics were included as variables in the model if, based on the literature, they were considered to be medically-related to vascular complications. These included: geographic region, age, sex, diabetes duration, BMI, $\mathrm{HbA}_{1 \mathrm{c}}$, FPG before breakfast, PPG after breakfast, TC, TG, creatinine (microvascular complications model only), HDL-C, LDL-C and SBP. Any significant correlates from this univariate logistical regression were subsequently entered into a stepwise multivariate logistical regression and only statistically significant correlates remained in the model. The statistical significance level employed was $\alpha=0.05$ (two tailed). P-values, odds ratio (OR) and 95\% confidence intervals (CIs) were calculated.

\section{Results}

\section{Baseline patient characteristics}

Baseline patient characteristics are summarised in Table 1. Of the 66,726 patients enrolled in $A_{1}$ chieve: 17,806 had macrovascular complications and 38,670 had no macrovascular complications at baseline; 35,078 had microvascular complications and 21,190 had no microvascular complications at baseline; and 18,420 had no macrovascular or microvascular complications at baseline. South Asia had the youngest patients with macrovascular complications (mean 56.3 years) and Latin America the oldest (mean 65.1 years). The Middle East had the youngest patients with microvascular complications (mean 54.6 years) and Latin America the oldest (mean 61.0 years; Table 1). The highest BMI among patients with macrovascular complications was recorded in Russia and the lowest in China (Table 1). The same pattern was seen in patients with microvascular complications, with the highest in Russia and the lowest in China (Table 1). In patients with macrovascular complications, the longest mean diabetes duration was in Latin America and North Africa and the shortest in South Asia. Mean diabetes duration in patients with microvascular complications was longest in Latin America and North Africa and the shortest in South Asia (Table 1).

\section{Metabolic control and QoL by geographical region}

Baseline $\mathrm{HbA}_{1 \mathrm{c}}$ and FPG values were similarly high across the different regions and in patients with macrovascular complications or microvascular complications (Table 2). Mean baseline $\mathrm{HbA}_{1 \mathrm{c}}$ values were $\geq 9.1 \%(\geq 76 \mathrm{mmol} / \mathrm{mol})$ in patients with macrovascular complications across all regions and $\geq 9.4 \%(\geq 79 \mathrm{mmol} / \mathrm{mol})$ across all regions in patients with microvascular complications. The baseline data for other measurements related to metabolic control and QoL are also displayed in Table 2.

\section{Complications by geographical region}

There were 17,806 patients with reported macrovascular complications and 35,078 with microvascular complications (Table 3). Macrovascular complications were reported in $27 \%$ of participants overall, with Russia having the highest percentage (72\%) and China the lowest (21\%) (Table 3). Microvascular complications were reported in 37 to $89 \%$ of participants, with Russia having the highest percentage and South Asia the lowest. Neuropathy was the highest reported microvascular complication in all regions, ranging from $25 \%$ in South Asia to $83 \%$ in Russia.

\section{Regression analysis}

The univariate logistical regression revealed that all correlates entered into both models were statistically significant (all $\mathrm{p}<0.05$ ). Subsequently, all correlates were entered into a stepwise multivariate logistical regression, and only statistically significant association remained in the models. After adjusting for regional differences, age, BMI, diabetes duration, TC, TG, LDL-C and SBP were positively correlated with macrovascular complications (all $\mathrm{p}<0.05$; Figure 1a). Female gender, HDL-C, FPG and $\mathrm{HbA}_{1 \mathrm{c}}$ were negatively correlated with macrovascular complications (all $\mathrm{p}<0.05$; Figure 1a). Age, BMI, diabetes duration, $\mathrm{HbA}_{1 \mathrm{c}}$, LDL-C, creatinine, and SBP were positively correlated with microvascular complications (all $\mathrm{p}<0.01$; Figure $1 \mathrm{~b}$ ). HDL-C levels were negatively correlated with microvascular complications ( $\mathrm{p}<0.001$; Figure $1 \mathrm{~b})$. 


\begin{tabular}{|c|c|c|c|c|c|c|c|c|}
\hline & All & China & South Asia & East Asia & North Africa & Middle East & Latin America & Russia \\
\hline \multicolumn{9}{|l|}{ All patients } \\
\hline \multicolumn{9}{|l|}{ Age, yr } \\
\hline $\mathrm{n}$ & 65,381 & 10,889 & 21,775 & 9,966 & 4,011 & 14,549 & 1,126 & 3,065 \\
\hline mean (SD) & $54.0(12.0)$ & $54.9(14.7)$ & $51.7(10.2)$ & $56.5(12.1)$ & $58.1(11.8)$ & $52.5(11.4)$ & $59.5(12.9)$ & $59.2(9.2)$ \\
\hline \multicolumn{9}{|l|}{ Sex } \\
\hline $\mathrm{n}$ & 66,656 & 11,019 & 22,436 & 10,030 & 4,032 & 14,927 & 1,138 & 3,074 \\
\hline male (\%) & $37,033(55.6)$ & $6,300(57.2)$ & $13,908(62.0)$ & $5,092(50.8)$ & $1,717(42.6)$ & $8,636(57.9)$ & $485(42.6)$ & $895(29.1)$ \\
\hline \multicolumn{9}{|l|}{$\mathrm{BMl}, \mathrm{kg} / \mathrm{m}^{2}$} \\
\hline $\mathrm{n}$ & 59,115 & 10,817 & 19,292 & 8,664 & 3,611 & 12,571 & 1,092 & 3,068 \\
\hline mean (SD) & $27.1(5.0)$ & $24.7(3.4)$ & $26.3(3.8)$ & $24.8(4.3)$ & $28.0(5.0)$ & $30.4(5.5)$ & $29.4(5.6)$ & $31.1(5.3)$ \\
\hline \multicolumn{9}{|c|}{ Diabetes duration, yr } \\
\hline$n$ & 65,786 & 10,814 & 22,316 & 9,776 & 4,016 & 14,658 & 1,135 & 3,071 \\
\hline mean (SD) & $8.0(6.2)$ & $6.3(6.3)$ & $6.4(4.7)$ & $8.6(6.9)$ & $11.5(7.2)$ & $9.8(6.0)$ & $12.0(8.3)$ & $8.4(5.4)$ \\
\hline \multicolumn{9}{|c|}{ Patients with macrovascular complications } \\
\hline \multicolumn{9}{|l|}{ Age, yr } \\
\hline $\mathrm{n}$ & 17,506 & 2,318 & 4,806 & 2,662 & 972 & 4,195 & 333 & 2,220 \\
\hline mean (SD) & $59.3(10.6)$ & $61.7(14.1)$ & $56.3(8.9)$ & $61.5(10.6)$ & $63.6(9.5)$ & $57.7(10.1)$ & $65.1(9.9)$ & $61.0(8.5)$ \\
\hline \multicolumn{9}{|l|}{ Sex } \\
\hline $\mathrm{n}$ & 17,786 & 2,342 & 4,943 & 2,684 & 978 & 4,278 & 335 & 2,226 \\
\hline male (\%) & $9,618(54.1)$ & $1,271(54.3)$ & $3,178(64.3)$ & $1,350(50.3)$ & $488(49.9)$ & $2,567(60.0)$ & $156(46.6)$ & $608(27.3)$ \\
\hline \multicolumn{9}{|l|}{$\mathrm{BMI}, \mathrm{kg} / \mathrm{m}^{2}$} \\
\hline$n$ & 16,271 & 2,306 & 4,645 & 2,310 & 867 & 3,603 & 317 & 2,223 \\
\hline mean (SD) & $27.9(5.3)$ & $25.1(3.4)$ & $26.2(3.6)$ & $25.3(4.3)$ & $28.8(5.1)$ & $31.1(5.5)$ & $30.4(6.0)$ & $31.6(5.3)$ \\
\hline \multicolumn{9}{|c|}{ Diabetes duration, yr } \\
\hline $\mathrm{n}$ & 17,653 & 2,297 & 4,938 & 2,635 & 973 & 4,251 & 335 & 2,224 \\
\hline mean (SD) & $10.3(6.6)$ & $9.4(7.0)$ & $8.4(5.1)$ & $10.5(7.3)$ & $14.2(7.9)$ & $12.4(6.4)$ & $14.6(8.6)$ & $9.2(5.4)$ \\
\hline \multicolumn{9}{|c|}{ Patients with microvascular complications } \\
\hline \multicolumn{9}{|l|}{ Age, yr } \\
\hline $\mathrm{n}$ & 34,518 & 5,399 & 8,103 & 5,582 & 2,386 & 9,602 & 710 & 2,736 \\
\hline mean (SD) & $56.9(11.2)$ & $57.9(14.1)$ & $54.8(9.7)$ & $59.0(11.2)$ & $60.9(10.5)$ & $54.6(10.4)$ & $61.0(12.1)$ & $59.8(8.9)$ \\
\hline \multicolumn{9}{|l|}{ Sex } \\
\hline $\mathrm{n}$ & 35,033 & 5,467 & 8,288 & 5,614 & 2,395 & 9,810 & 715 & 2,744 \\
\hline male (\%) & $18,542(52.9)$ & $2,957(54.1)$ & $5,067(61.1)$ & $2,752(49.0)$ & $994(41.5)$ & $5,703(58.1)$ & $293(41.0)$ & $776(28.3)$ \\
\hline \multicolumn{9}{|l|}{$\mathrm{BMI}, \mathrm{kg} / \mathrm{m}^{2}$} \\
\hline $\mathrm{n}$ & 31,894 & 5,364 & 7,644 & 4,946 & 2,154 & 8,361 & 686 & 2,739 \\
\hline mean (SD) & $27.6(5.3)$ & $24.7(3.4)$ & $26.1(3.7)$ & $25.0(4.3)$ & $28.4(5.0)$ & $30.9(5.5)$ & $29.4(5.7)$ & $31.2(5.3)$ \\
\hline \multicolumn{9}{|c|}{ Diabetes duration, yr } \\
\hline $\mathrm{n}$ & 34,666 & 5,371 & 8,281 & 5,514 & 2,379 & 9,667 & 712 & 2,742 \\
\hline mean (SD) & $9.9(6.4)$ & $8.4(6.7)$ & $8.2(4.9)$ & $10.4(7.2)$ & $13.6(7.3)$ & $11.2(6.0)$ & $13.7(8.3)$ & $8.9(5.4)$ \\
\hline
\end{tabular}

Note: due to the observational nature of this study, not all baseline data were recorded.

$\mathrm{BMI}=$ body mass index.

Use of vascular disease preventative drugs by geographical region

Among participants with macrovascular complications overall, $67 \%$ reported taking statins, while the proportion was 56\% among participants with microvascular complications. For participants with macrovascular complications and those with microvascular complications, the highest statin use was in the Middle East (86\% and 74\%, 
Table 2 Baseline metabolic control and quality of life by geographical region

\begin{tabular}{|c|c|c|c|c|c|c|c|c|}
\hline & All & China & South Asia & East Asia & North Africa & Middle East & Latin America & Russia \\
\hline \multicolumn{9}{|c|}{$\begin{array}{l}\text { Patients with macrovascular } \\
\text { complications }\end{array}$} \\
\hline \multicolumn{9}{|l|}{$\mathrm{HbA}_{1 c} \%$} \\
\hline $\mathrm{n}$ & 12,727 & 1,332 & 4,002 & 1,170 & 620 & 3,426 & 147 & 2,030 \\
\hline mean (SD) & $9.5(1.7)$ & $9.1(2.1)$ & $9.3(1.2)$ & $9.7(2.0)$ & $9.6(1.8)$ & $9.7(1.7)$ & $9.6(2.4)$ & $9.6(1.7)$ \\
\hline \multicolumn{9}{|l|}{$\mathrm{HbA}{ }_{1 c}, \mathrm{mmol} / \mathrm{mol}$} \\
\hline $\mathrm{n}$ & 12,727 & 1,332 & 4,002 & 1,170 & 620 & 3,426 & 147 & 2,030 \\
\hline mean (SD) & 80.3 (18.6) & $76.0(23.0)$ & $78.2(13.1)$ & $82.5(21.9)$ & $81.4(19.7)$ & 82.5 (18.6) & $81.4(26.2)$ & $81.4(18.6)$ \\
\hline \multicolumn{9}{|l|}{ FPG before breakfast } \\
\hline $\mathrm{n}$ & 13,603 & 1,859 & 4,064 & 1,431 & 715 & 3,143 & 204 & 2,187 \\
\hline mmol/l, mean (SD) & $10.7(3.3)$ & $9.8(3.6)$ & $10.8(2.8)$ & $11.2(4.2)$ & $11.2(4.2)$ & $11.0(3.3)$ & $10.7(4.2)$ & $10.4(2.6)$ \\
\hline mg/dl, mean (SD) & $192.8(59.6)$ & $176.5(64.0)$ & $194.2(50.5)$ & $202.4(74.9)$ & $201.3(75.7)$ & $198.0(60.1)$ & $191.9(76.5)$ & $187.7(46.9)$ \\
\hline \multicolumn{9}{|l|}{ PPG after breakfast } \\
\hline $\mathrm{n}$ & 9,704 & 1,451 & 2,984 & 784 & 376 & 2,254 & 30 & 1,825 \\
\hline mmol/l, mean (SD) & $14.5(4.3)$ & $14.0(4.7)$ & $15.3(3.7)$ & $15.4(4.9)$ & $14.7(5.2)$ & $15.4(4.4)$ & $13.1(5.9)$ & $12.2(3.1)$ \\
\hline mg/dl, mean (SD) & $262.0(77.0)$ & $252.1(85.4)$ & $275.9(65.9)$ & $277.0(87.6)$ & $265.5(94.0)$ & $278.1(78.9)$ & $235.1(105.6)$ & $220.3(55.6)$ \\
\hline \multicolumn{9}{|c|}{ Total cholesterol, mmol// } \\
\hline $\mathrm{n}$ & 7,176 & 851 & 466 & 744 & 378 & 2,530 & 136 & 2,071 \\
\hline mean (SD) & $5.5(1.3)$ & $5.1(1.2)$ & $5.2(0.8)$ & $5.3(1.5)$ & $4.6(1.3)$ & $5.4(1.2)$ & $5.6(1.7)$ & $6.1(1.3)$ \\
\hline \multicolumn{9}{|l|}{ Triglycerides, mmol// } \\
\hline $\mathrm{n}$ & 6,732 & 844 & 703 & 637 & 402 & 2,524 & 116 & 1,506 \\
\hline mean (SD) & $2.1(1.0)$ & $2.0(1.3)$ & $2.2(0.7)$ & $2.0(1.0)$ & $1.6(0.9)$ & $2.3(1.0)$ & $2.4(1.2)$ & $2.1(1.0)$ \\
\hline \multicolumn{9}{|l|}{ Creatinine, $\mu \mathrm{mol} / \mathrm{l}$} \\
\hline$n$ & 6,865 & 724 & 776 & 627 & 350 & 2,370 & 103 & 1,915 \\
\hline mean (SD) & $87.7(32.9)$ & $48.7(43.6)$ & $96.2(30.3)$ & $97.6(35.1)$ & $89.5(29.6)$ & $95.7(29.4)$ & $94.5(28.6)$ & $85.2(19.5)$ \\
\hline \multicolumn{9}{|l|}{$\mathrm{HDL}-\mathrm{C}, \mathrm{mmol} / \mathrm{l}$} \\
\hline $\mathrm{n}$ & 5,602 & 777 & 714 & 539 & 255 & 2,283 & 98 & 936 \\
\hline mean (SD) & $1.1(0.4)$ & $1.2(0.4)$ & $1.0(0.2)$ & $1.2(0.5)$ & $1.1(0.4)$ & $1.0(0.3)$ & $1.0(0.3)$ & $1.4(0.6)$ \\
\hline \multicolumn{9}{|l|}{ LDL-C, mmol/l } \\
\hline $\mathrm{n}$ & 5,711 & 794 & 713 & 545 & 238 & 2,364 & 94 & 963 \\
\hline mean (SD) & $3.2(1.1)$ & $3.0(1.0)$ & $3.4(1.0)$ & $3.4(1.3)$ & $2.8(1.4)$ & $3.2(1.0)$ & $3.2(1.1)$ & $3.4(1.2)$ \\
\hline \multicolumn{9}{|l|}{$\mathrm{SBP}, \mathrm{mmHg}$} \\
\hline $\mathrm{n}$ & 13,744 & 1,552 & 3,376 & 1,969 & 755 & 3,619 & 274 & 2,199 \\
\hline mean (SD) & $141.0(19.5)$ & $137.2(18.3)$ & $145.2(21.0)$ & $133.3(18.7)$ & $137.8(20.0)$ & $141.4(18.9)$ & $131.8(16.5)$ & $145.7(16.5)$ \\
\hline \multicolumn{9}{|l|}{ QoL* $^{*}$} \\
\hline $\mathrm{n}$ & 11,329 & 1,712 & 3,611 & 2,156 & 676 & 865 & 261 & 2,048 \\
\hline mean (SD) & $60.2(16.5)$ & 74.4 (13.9) & $52.7(10.6)$ & $66.7(15.7)$ & $59.4(17.8)$ & $62.1(14.9)$ & 63.7 (17.8) & $53.5(16.9)$ \\
\hline \multicolumn{9}{|c|}{$\begin{array}{l}\text { Patients with microvascular } \\
\text { complications }\end{array}$} \\
\hline \multicolumn{9}{|l|}{$\mathrm{HbA}_{1 c}, \%$} \\
\hline$n$ & 24,352 & 3,128 & 6,673 & 2,385 & 1,545 & 7,726 & 378 & 2,517 \\
\hline mean (SD) & $9.6(1.7)$ & $9.4(2.3)$ & $9.4(1.3)$ & $9.7(2.0)$ & $9.5(1.8)$ & $9.7(1.7)$ & $10.1(2.2)$ & $9.6(1.7)$ \\
\hline \multicolumn{9}{|l|}{$\mathrm{HbA}{ }_{1 c}, \mathrm{mmol} / \mathrm{mol}$} \\
\hline $\mathrm{n}$ & 24,352 & 3,128 & 6,673 & 2,385 & 1,545 & 7,726 & 378 & 2,517 \\
\hline mean (SD) & 81.4 (18.6) & $79.2(25.1)$ & $79.2(14.2)$ & 82.5 (21.9) & $80.3(19.7)$ & 82.5 (18.6) & $86.9(24.0)$ & $81.4(18.6)$ \\
\hline
\end{tabular}


Table 2 Baseline metabolic control and quality of life by geographical region (Continued)

\begin{tabular}{|c|c|c|c|c|c|c|c|c|}
\hline \multicolumn{9}{|l|}{ FPG before breakfast } \\
\hline $\mathrm{n}$ & 25,986 & 4,239 & 6,655 & 3,015 & 1,744 & 7,162 & 480 & 2,691 \\
\hline mmol/l, mean (SD) & $10.8(3.5)$ & $10.0(3.5)$ & $10.7(3.0)$ & $11.3(4.2)$ & $11.1(4.1)$ & $11.2(3.5)$ & $11.7(4.6)$ & $10.4(2.7)$ \\
\hline mg/dl, mean (SD) & $194.5(62.9)$ & $179.4(63.6)$ & $193.3(54.3)$ & $202.8(75.5)$ & $200.4(74.5)$ & $201.5(62.4)$ & $210.5(83.3)$ & $186.9(47.7)$ \\
\hline \multicolumn{9}{|l|}{ PPG after breakfast } \\
\hline $\mathrm{n}$ & 18,300 & 3,297 & 4,762 & 1,657 & 1,025 & 5,183 & 110 & 2,266 \\
\hline mmol/l, mean (SD) & $14.8(4.5)$ & $13.8(4.8)$ & $15.5(3.9)$ & $15.6(5.0)$ & $14.8(4.7)$ & $15.5(4.4)$ & $15.6(6.0)$ & $12.1(3.0)$ \\
\hline mg/dl, mean (SD) & $265.8(80.3)$ & $249.4(86.7)$ & $279.0(69.7)$ & $281.5(89.3)$ & $265.7(84.6)$ & $279.6(80.1)$ & $281.4(107.3)$ & $218.3(54.9)$ \\
\hline \multicolumn{9}{|l|}{ Total cholesterol, mmol// } \\
\hline$n$ & 13,795 & 2,005 & 812 & 1,509 & 993 & 5,590 & 334 & 2,552 \\
\hline mean (SD) & $5.4(1.3)$ & $5.1(1.3)$ & $5.1(0.8)$ & $5.3(1.4)$ & $4.7(1.3)$ & $5.3(1.2)$ & $5.8(1.6)$ & $6.0(1.3)$ \\
\hline \multicolumn{9}{|l|}{ Triglycerides, mmol// } \\
\hline $\mathrm{n}$ & 13,356 & 1,970 & 1,371 & 1,257 & 1,030 & 5,572 & 290 & 1,866 \\
\hline mean (SD) & $2.1(1.1)$ & $2.1(1.3)$ & $2.1(0.8)$ & $2.0(1.1)$ & $1.6(0.9)$ & $2.2(1.0)$ & $2.5(1.3)$ & $2.1(1.0)$ \\
\hline \multicolumn{9}{|l|}{ Creatinine, $\mu \mathrm{mol} / \mathrm{l}$} \\
\hline $\mathrm{n}$ & 13,399 & 1,779 & 1,482 & 1,185 & 893 & 5,440 & 261 & 2,359 \\
\hline mean (SD) & $83.5(32.7)$ & $52.3(42.3)$ & 89.9 (27.9) & 95.8 (37.9) & $85.0(28.2)$ & $88.2(28.6)$ & $89.2(26.4)$ & $84.8(19.1)$ \\
\hline \multicolumn{9}{|l|}{$\mathrm{HDL}-\mathrm{C}, \mathrm{mmol} / \mathrm{l}$} \\
\hline$n$ & 11,472 & 1,823 & 1,398 & 1,083 & 678 & 5,067 & 234 & 1,189 \\
\hline mean (SD) & $1.1(0.4)$ & $1.2(0.4)$ & $1.0(0.2)$ & $1.2(0.4)$ & $1.1(0.4)$ & $1.1(0.3)$ & $1.0(0.3)$ & $1.4(0.6)$ \\
\hline \multicolumn{9}{|l|}{ LDL-C, mmol// } \\
\hline $\mathrm{n}$ & 11,580 & 1,852 & 1,379 & 1,090 & 646 & 5,185 & 217 & 1,211 \\
\hline mean (SD) & $3.2(1.1)$ & $3.1(1.1)$ & $3.1(0.9)$ & $3.2(1.2)$ & $2.9(1.4)$ & $3.2(1.0)$ & $3.2(1.1)$ & $3.3(1.1)$ \\
\hline \multicolumn{9}{|l|}{$\mathrm{SBP}, \mathrm{mmHg}$} \\
\hline $\mathrm{n}$ & 26,661 & 3,487 & 5,656 & 4,022 & 1,899 & 8,282 & 614 & 2,701 \\
\hline mean (SD) & $137.3(18.7)$ & $134.9(17.5)$ & $141.4(21.0)$ & $132.0(17.9)$ & $135.2(18.8)$ & $137.1(17.4)$ & $131.5(17.2)$ & $143.1(16.8)$ \\
\hline \multicolumn{9}{|l|}{ QoL $^{*}$} \\
\hline $\mathrm{n}$ & 21,166 & 3,947 & 6,103 & 4,489 & 1,741 & 1,805 & 555 & 2,526 \\
\hline mean (SD) & $62.3(16.9)$ & 75.2 (13.8) & $52.8(11.1)$ & $67.9(16.0)$ & $61.1(17.5)$ & $64.1(15.5)$ & $64.8(18.3)$ & $54.3(17.0)$ \\
\hline
\end{tabular}

Note: due to the observational nature of this study, not all baseline data were recorded.

${ }^{*} \mathrm{EQ}-5 \mathrm{D}^{\mathrm{TM}}$ (100 = best imaginable health; $0=$ worst imaginable health) [20].

Table 3 Baseline patient complications by geographical region

\begin{tabular}{lrrrrrrrr}
\hline & All & China & South Asia & East Asia & North Africa & Middle East & Latin America & Russia \\
\hline $\begin{array}{l}\text { Patients with macrovascular } \\
\text { complications, n (\%) }\end{array}$ & $17,806(27.2)$ & $2,342(21.3)$ & $4,946(23.3)$ & $2,685(26.8)$ & $979(24.2)$ & $4,293(28.7)$ & $335(29.4)$ & $2,226(72.4)$ \\
$\begin{array}{l}\text { Patients with microvascular } \\
\text { complications, n (\%) }\end{array}$ & $35,078(53.5)$ & $5,467(49.6)$ & $8,293(39.0)$ & $5,615(56.0)$ & $2,397(59.4)$ & $9,847(65.8)$ & $715(62.8)$ & $2,744(89.3)$ \\
Renal disease, n (\%) & $18,271(27.9)$ & $2,455(22.3)$ & $4,321(20.3)$ & $2,845(28.4)$ & $1,077(26.7)$ & $6,108(40.8)$ & $376(33.0)$ & $1,089(35.4)$ \\
Eye problems, n (\%) & $17,198(26.3)$ & $2,430(22.1)$ & $3,464(16.3)$ & $2,380(23.7)$ & $1,354(33.5)$ & $5,081(33.9)$ & $368(32.3)$ & $2,121(69.0)$ \\
Foot ulcer, n (\%) & $3,538(5.4)$ & $274(2.5)$ & $1,046(4.9)$ & $536(5.3)$ & $147(3.6)$ & $1,289(8.6)$ & $85(7.5)$ & $161(5.2)$ \\
Neuropathy, n (\%) & $25,179(38.4)$ & $3,671(33.3)$ & $5,234(24.6)$ & $3,706(36.9)$ & $1,530(37.9)$ & $7,995(53.4)$ & $491(43.1)$ & $2,552(83.0)$ \\
\hline
\end{tabular}

Note: due to the observational nature of this study, not all baseline data were recorded. 


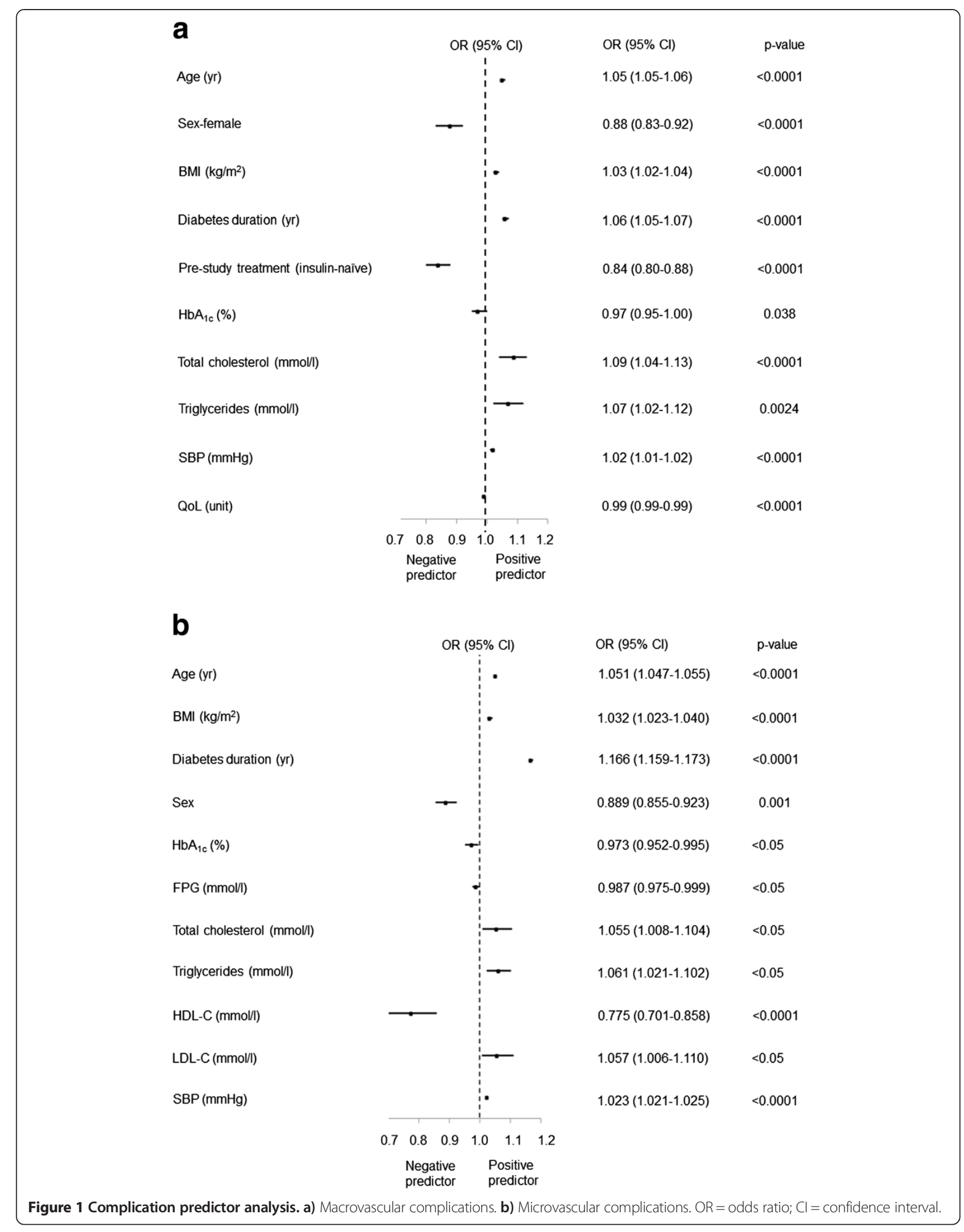


respectively) and the lowest was in China (49\% and 34\%, respectively). Use of vascular disease preventative drugs was higher among participants with macrovascular complications (statins 67\%, aspirin 79\%, RAS blocker 78\%) than those with microvascular complications (statins 56\%, aspirin 64\%, RAS blocker 63\%) (Table 4).

In all regions, aspirin use was higher in participants with macrovascular complications than those with microvascular complications (Table 4). In addition, aspirin use was higher than statin use in all regions except East Asia, where usage levels of the two drugs were similar among participants with macrovascular complications, and statin use was higher than aspirin use among participants with microvascular complications (Table 4).

A higher proportion of participants with macrovascular complications used a RAS blocker than those with microvascular complications in all regions apart from Russia, where usage was similar for the two groups. Russia also had the highest use of RAS blocker, both among participants with macrovascular complications (93\%) and those with microvascular complications (87\%). In contrast, use of RAS blocker was lowest in China for both participant groups (56\% of participants with macrovascular complications and $42 \%$ of participants with microvascular complications).

\section{Discussion}

Baseline $\mathrm{HbA}_{1 \mathrm{c}}$ was high across all geographical regions, and vascular complication rates were generally high. In addition, the use of vascular protective therapies, even in those patients with vascular complications, was suboptimal. The particularly low reported use of vascular protective therapies in patients from China is commensurate with previous findings [21]. The findings suggest poor glycaemic control and suboptimal diabetes management across many geographical regions, which may be due to poor adherence to treatment regimens, lack of access to therapy, poor diet, and delay in initiating, or failure to adequately optimise, insulin therapy [12-14,16,22]. For example, significant numbers of people with diabetes have poor psychological wellbeing and these psychological problems can adversely affect adherence to treatment [22]. Some of the variation seen in the prevalence of complications in different geographical regions may have been due to variations in clinical care, healthcare resources, diagnostic criteria, and definitions used in routine clinical practice, among other reasons. In particular, the high rates of complications among participants from Russia may have been due to a variety of factors, such as late diagnoses of diabetes, late initiation of treatment, or lifestyle factors. Without large-scale epidemiological studies, it is difficult to ascertain the extent to which clinical practice may impact on the rate of complications in people with diabetes in Russia or other regions.

Results of the correlation analysis revealed relationships between vascular complications and various disease characteristics. Age, BMI, diabetes duration, LDL-C, and SBP were positively associated with macrovascular and microvascular complications, while TC and TG were positively correlated with macrovascular complications and creatinine with microvascular complications. These findings are consistent with the large evidence base showing that the risk of developing these complications is positively associated with these variables [23-27]. The negative association between HDL-C values and macrovascular and microvascular complications would also be expected given the documented inverse relationship between blood levels of HDL-C and cardiovascular disease risk [28]. The profile of cholesterol levels is important in people with type 2 diabetes as it reflects atherogenic dyslipidaemia, which is characterised by small dense LDL-C, low HDL-C and high TG levels [29]. Regarding the result suggesting female gender was associated with a lower frequency of macrovascular

Table 4 Use of vascular disease preventative drugs by geographical region

\begin{tabular}{|c|c|c|c|c|c|c|c|c|}
\hline & All & China & South Asia & East Asia & North Africa & Middle East & Latin America & Russia \\
\hline \multicolumn{9}{|c|}{$\begin{array}{l}\text { Patients with macrovascular } \\
\text { complications }\end{array}$} \\
\hline RAS blocker, n (\%) & $13,910(78.1)$ & 1,303 (55.6) & $3,771(76.3)$ & 1,992 (74.2) & 778 (79.5) & 3,719 (86.6) & $285(85.1)$ & 2,062 (92.6) \\
\hline Aspirin, n (\%) & 13,995 (78.6) & $1,644(70.2)$ & $3,784(76.6)$ & $1,866(69.5)$ & 801 (81.8) & $3,973(92.5)$ & $252(75.2)$ & $1,675(75.2)$ \\
\hline Statins, n (\%) & $11,958(67.2)$ & 1,139 (48.6) & $3,057(61.9)$ & $1,846(68.8)$ & $715(73.0)$ & $3,710(86.4)$ & $189(56.4)$ & $1,302(58.5)$ \\
\hline \multicolumn{9}{|c|}{$\begin{array}{l}\text { Patients with microvascular } \\
\text { complications }\end{array}$} \\
\hline RAS blocker, n (\%) & $21,884(62.6)$ & 2,268 (41.5) & 4,792 (58.5) & $3,465(61.7)$ & $1,557(65.0)$ & $6,907(70.1)$ & 499 (69.8) & 2,396 (87.3) \\
\hline Aspirin, n (\%) & $22,207(63.5)$ & $2,704(49.5)$ & 4,958 (60.6) & 2,698 (48.0) & $1,598(66.7)$ & $7,922(80.5)$ & $427(59.7)$ & $1,900(69.2)$ \\
\hline Statins, n (\%) & $19,709(56.4)$ & $1,856(33.9)$ & 4,325 (52.8) & 3,133 (55.8) & $1,288(53.7)$ & $7,323(74.4)$ & $312(43.6)$ & $1,472(53.6)$ \\
\hline
\end{tabular}

Note: due to the observational nature of this study, not all baseline data were recorded.

RAS = renin-angiotensin system 
complications, past research in this area has shown mixed results [30-32].

In contrast, the finding that $\mathrm{HbA}_{1 \mathrm{c}}$ and $\mathrm{FPG}$ values were negatively associated with establishment of macrovascular complications was surprising. The correlation coefficient between $\mathrm{HbA}_{1 \mathrm{c}}$ and FPG values was $\mathrm{r}=0.44$, and therefore, colineairty is not likely to have distorted the model and explain this finding. One possible explanation for this surprising result is the study design. The $A_{1}$ chieve study was a cross-sectional study and as such, the present baseline $\mathrm{HbA}_{1 \mathrm{c}}$ and FPG values were used to predict the establishment of diabetes complications which had developed over the course of the disease. However, there may not be a clear association between the present value and the value several years prior to this. In addition, complications (especially macrovascular complications) usually take several years to develop in the presence of high $\mathrm{HbA}_{1 \mathrm{c}}$ levels, and so an association would become apparent in a cohort study rather than a cross-sectional study. The temporal relationships between $\mathrm{HbA}_{1 \mathrm{c}}$ levels and establishment of macrovascular and/or microvascular complications must be further established before fully understanding the power of current $\mathrm{HbA}_{1 \mathrm{c}}$ levels to predict complications.

Alternative explanations for this unusual finding could be the fact that the study population included a very heterogenous group ranging across four continents, leading to genetic variability, dietary variability and risk factor variability. Minimising these confounding factors may have provided a clearer picture of the relationships between the various disease parameters and vascular complications and this could be an area for future research. In addition, there were no specific or defined measurements of the macrovascular and microvascular complications reported in this study, and these were classified based on clinical judgement of the physicians. Other limitations of this observational study include potential bias and lack of a control group; however, an advantage of this observational study was that it had less stringent inclusion and exclusion criteria, allowing very large numbers of people to be assessed in many different geographical regions, something that is virtually impossible with randomised controlled trials.

\section{Conclusions}

Across all the geographical regions, patients showed poor glycaemic control at the point of commencing on insulin analogues, vascular complication rates were generally high, and use of vascular disease preventative therapies was generally sub-optimal. Many of the disease characteristics were statistically significantly associated with macrovascular and/or microvascular complications at baseline. These findings suggest that suboptimal diabetes therapy is contributing to the burden of type 2 diabetes worldwide. Closer adherence to diabetes guidelines, which may include earlier initiation and optimisation of insulin regimens (e.g., with insulin analogues in the $\mathrm{A}_{1}$ chieve population), may reduce the prevalence of vascular complications, improve the lives of people with diabetes and reduce the burden on healthcare systems.

\section{Consent}

Participants gave written informed consent for the results of the study to be published in a scientific or medical journal.

\section{Abbreviations \\ ADA: American Diabetes Association; BMl: Body mass index; Cls: 95\% confidence intervals; EASD: European Association for the Study of Diabetes; EQ-5D: European questionnaire - 5 dimensions; FPG: Fasting plasma glucose; $\mathrm{HbA}_{1 \mathrm{c}}$ : Glycated haemoglobin; HDL-C: High-density lipoprotein-cholesterol; IDF: International Diabetes Federation; LDL-C: Low-density lipoprotein- cholesterol; mg/dl: Milligram per decilitre; $\mathrm{mm} / \mathrm{Hg}$ : Millimetres of mercury; mmol/l: Millimoles per litre; Ml: Myocardial infarction; n: Number; OR: Odds ratio; OGLDs: Oral glucose-lowering drugs; P: Probability; PPG: Post-prandial plasma glucose; QoL: Quality of life; RAS: Renin-angiotensin system; SADRs: Serious adverse drug reactions; SD: Standard deviation; SBP: Systolic blood pressure; TC: Total cholesterol; TG: Triglycerides; UKPDS: United Kingdom Prospective Diabetes Study.}

\section{Competing interests}

LL is a speaker and member of the Latin American Board of Eli Lilly, and the Argentinean Boards of Novo Nordisk, Novartis, Sanofi, BMS, and Astra Zeneca. He is also a principal investigator of clinical trials run by Eli Lilly, Novo Nordisk, and Astra Zeneca. SYG and her affiliated institution have received funding from Novo Nordisk and Sanofi for research, advisory and educational activities. ZH has received funding from Novo Nordisk, Eli Lilly and Sanofi for research, advisory and education activities. RM has participated in several advisory boards for Novo Nordisk and has participated in studies sponsored by Novo Nordisk. He has also participated in symposia run by Novo Nordisk, Sanofi, Eli Lilly, Merck, and Pfizer. VP is an employee of Novo Nordisk A/S. MEK is a principal investigator of a clinical trial in Iran run by Novo Nordisk A/S and also an observational study supported by Sanofi.

\section{Authors' contributions}

$L L, S Y G, Z H, R M, V P$ and MEK were all involved in the interpretation of data, involved in drafting the manuscript or revising it critically for important intellectual content and have given final approval of the version of the manuscript to be published.

\section{Acknowledgements}

The $A_{1}$ chieve study and this manuscript were funded by Novo Nordisk A/S (Bagsvaerd, Denmark). Editorial assistance on this manuscript was provided by Martin Gilmour and John Clarke at ESP Bioscience (Crowthorne, UK), funded by Novo Nordisk A/S.

\section{Author details}

${ }^{1}$ Endocrinology and Nuclear Medicine Unit, Diabetes and Metabolism Section, Hospital Italiano de Buenos Aires, Buenos Aires, Argentina. ${ }^{2}$ Department of Endocrinology, Singapore General Hospital, Singapore. ${ }^{3}$ Medical Department, Hospital Putrajaya, Putrajaya, Malaysia. ${ }^{4}$ Department of Internal Medicine, CHU Sétif, Sétif, Algeria. ${ }^{5}$ Novo Nordisk A/S, Zürich, Switzerland. ${ }^{6}$ Endocrine Research Centre (Firouzgar), Institute of Endocrinology \& Metabolism, Tehran University of Medical Sciences, Tehran, Iran.

Received: 26 June 2013 Accepted: 27 September 2013 Published: 24 October 2013

\section{References}

1. Stratton IM, Adler Al, Neil HA, Matthews DR, Manley SE, Cull CA, Hadden D, Turner RC, Holman RR: Association of glycaemia with macrovascular and microvascular complications of type 2 diabetes (UKPDS 35): prospective observational study. BMJ 2000, 321:405-412. 
2. Holman RR, Paul SK, Bethel MA, Matthews DR, Neil HA: 10-year follow-up of intensive glucose control in type 2 diabetes. N Engl J Med 2008, 359:1577-1589.

3. International Diabetes Federation Clinical Guidelines Task Force: Global Guidelines for Type 2 Diabetes; 2012. http://www.idf.org/sites/default/files/ IDF-Guideline-for-Type-2-Diabetes.pdf.

4. Inzucchi SE, Bergenstal RM, Buse JB, Diamant M, Ferrannini E, Nauck M, Peters AL, Tsapas A, Wender R, Matthews DR: Management of hyperglycemia in type 2 diabetes: a patient-centered approach: position statement of the American Diabetes Association (ADA) and the European Association for the Study of Diabetes (EASD). Diabetes Care 2012, 35:1364-1379.

5. International Diabetes Federation: Guideline for Management of PostMeal Glucose in Diabetes; 2011. http://www.idf.org/sites/default/files/postmeal\% 20glucose\%20guidelines.pdf.

6. National Institute for Health and Clinical Excellence: National Institute for Health and Clinical Excellence (NICE) Guidelines. Type 2 diabetes: The Management of Type 2 Diabetes. London: Royal College of Physicians; 2008

7. Currie CJ, Peters JR, Tynan A, Evans M, Heine RJ, Bracco OL, Zagar T, Poole $\mathrm{CD}$ : Survival as a function of $\mathrm{HbA}(1 \mathrm{c})$ in people with type 2 diabetes: a retrospective cohort study. Lancet 2010, 375:481-489.

8. Saaddine JB, Cadwell B, Gregg EW, Engelgau MM, Vinicor F, Imperatore G, Narayan KM: Improvements in diabetes processes of care and intermediate outcomes: United States, 1988-2002. Ann Intern Med 2006, 144:465-474.

9. Saydah SH, Fradkin J, Cowie CC: Poor control of risk factors for vascular disease among adults with previously diagnosed diabetes. JAMA 2004 291:335-342.

10. Vaag A, Lund SS: Insulin initiation in patients with type 2 diabetes mellitus: treatment guidelines, clinical evidence and patterns of use of basal vs premixed insulin analogues. Eur J Endocrinol 2012, 166:159-170.

11. Chan JC, Gagliardino JJ, Baik SH, Chantelot JM, Ferreira SR, Hancu N, Ilkova $H$, Ramachandran A, Aschner P, IDMPS Investigators: Multifaceted determinants for achieving glycemic control: the International Diabetes Management Practice Study (IDMPS). Diabetes Care 2009, 32:227-233.

12. Delamater AM: Improving patient adherence. Clinical Diabetes 2006 24:71-77.

13. Elgrably F, Costagliola D, Chwalow AJ, Varenne P, Slama G, Tchobroutsky G: Initiation of insulin treatment after 70 years of age: patient status 2 years later. Diabet Med 1991, 8:773-777.

14. Korytkowski M: When oral agents fail: practical barriers to starting insulin. Int J Obes Relat Metab Disord 2005, 26(Suppl 3):S18-24

15. Peyrot M, Rubin RR, Lauritzen T, Snoek FJ, Matthews DR, Skovlund SE: Psychosocial problems and barriers to improved diabetes management: results of the cross-national Diabetes Attitudes, Wishes and Needs (DAWN) Study. Diabet Med 2005, 22:1379-1385.

16. Raman R, Gupta A, Kulothungan V, Sharma T: Prevalence and risk factors of diabetic retinopathy in subjects with suboptimal glycemic, blood pressure and lipid control. Sankara Nethralaya Diabetic Retinopathy Epidemiology and Molecular Genetic Study (SN-DREAMS, Report 33). Curr Eye Res 2012, 37:513-523.

17. Home P, Naggar NE, Khamseh M, Gonzalez-Galvez G, Shen C, Chakkarwar P, Wenying Y: An observational non-interventional study of people with diabetes beginning or changed to insulin analogue therapy in nonWestern countries: the $A_{1}$ chieve study. Diabetes Res Clin Pract 2011 94:352-363

18. World Medical Association: Declaration of Helsinki - Ethical Principles for Medical Research involving Human Subjects; 2008. http://www.riip-influenza. org/wp-content/uploads/2011/08/Helsinki-2008-EN.pdf.

19. International Society for Pharmacoepidemiology: Guidelines for Good Pharmacoepidemiology Practices (GPP). 2007. Revision 2, 2007. http://www. pharmacoepi.org/resources/guidelines_08027.cfm.

20. EuroQol Group: EuroQol - a new facility for the measurement of healthrelated quality of life. Health Policy 1990, 16:199-208.

21. Li HJ, Liu J, Liu J, Wang W, Qin LP, Li Y, Wang M, Sun JY, Qi Y, Zhao D: Real world use of statins for secondary prevention in patients with high risk coronary heart disease in China. Zhonghua Xin Xue Guan Bing Za Zhi 2010, 38:1033-1037.

22. Peyrot M, Rubin RR, Lauritzen T, Skovlund SE, Snoek FJ, Matthews DR, Landgraf R, Kleinebreil L: Resistance to insulin therapy among patients and providers: results of the cross-national Diabetes Attitudes, Wishes, and Needs (DAWN) study. Diabetes Care 2005, 28:2673-2679.

23. Moore DJ, Gregory JM, Kumah-Crystal YA, Simmons JH: Mitigating microand macro-vascular complications of diabetes beginning in adolescence. Vasc Health Risk Manag 2009, 5:1015-1031.

24. Stamler J, Vaccaro O, Neaton JD, Wentworth D: Diabetes, other risk factors, and 12-yr cardiovascular mortality for men screened in the Multiple Risk Factor Intervention Trial. Diabetes Care 1993, 16:434-444.

25. Tesfaye S, Chaturvedi N, Eaton SE, Ward JD, Manes C, lonescu-Tirgoviste C, Witte DR, Fuller JH: EURODIAB Prospective Complications Study Group. Vascular risk factors and diabetic neuropathy. N Engl J Med 2005, 352:341-350.

26. Wandell PE: Risk factors for microvascular and macrovascular complications in men and women with type 2 diabetes. Scand J Prim Health Care 1999, 17:116-121.

27. Zoppini G, Negri C, Stoico V, Casati S, Pichiri I, Bonora E: Triglyceride-highdensity lipoprotein cholesterol is associated with microvascular complications in type 2 diabetes mellitus. Metabolism 2012, 61:22-29.

28. Toth P: The 'good cholesterol': high density lipoprotein. Circulation 2005, 111:e89-91.

29. Krauss RM, Siri PW: Dyslipidemia in type 2 diabetes. Med Clin North Am 2004, 88:897-909.

30. Gorodeski G: Update on cardiovascular disease in post-menopausal women. Best Pract Res Clin Obstet Gynaecol 2002, 16:329-355.

31. Hayashi T, Kawashima S, Nomura H, Itoh H, Watanabe H, Ohrui T, Yokote K, Sone H, Hattori Y, Yoshizumi M, Ina K, Kubota K, Japan Cholesterol and Diabetes Mellitus Investigation Group: Age, gender, insulin and blood glucose control status alter the risk of ischemic heart disease and stroke among elderly diabetic patients. Cardiovasc Diabetol 2011, 10:86.

32. Awa W, Fach E, Krakow D, Weilp R, Kunder J, Voll A, Zeyfang A, Wagner C, Schutt M, Boehm B, de Souza M, Holl R, DPV Initiative, German BMBF Competence Networks Diabetes Mellitus and Obesity: Type 2 diabetes from pediatric to geriatric age: analysis of gender and obesity among 120-183 patients from the German/Austrian DPV database. Eur J Endocrinol 2012, 167:245-254

doi:10.1186/1758-5996-5-57

Cite this article as: Litwak et al:: Prevalence of diabetes complications in people with type 2 diabetes mellitus and its association with baseline characteristics in the multinational $\mathrm{A}_{1}$ chieve study. Diabetology \& Metabolic Syndrome 2013 5:57.

\section{Submit your next manuscript to BioMed Central and take full advantage of:}

- Convenient online submission

- Thorough peer review

- No space constraints or color figure charges

- Immediate publication on acceptance

- Inclusion in PubMed, CAS, Scopus and Google Scholar

- Research which is freely available for redistribution 